Bull. Korean Math. Soc. 46 (2009), No. 5, pp. 905-915

DOI 10.4134/BKMS.2009.46.5.905

\title{
ON CERTAIN CLASSES OF MULTIVALENT \\ FUNCTIONS INVOLVING A GENERALIZED \\ DIFFERENTIAL OPERATOR
}

\author{
Chellian Selvaraj and Kuppathai A. Selvakumaran
}

\begin{abstract}
Making use of a generalized differential operator we introduce some new subclasses of multivalent analytic functions in the open unit disk and investigate their inclusion relationships. Some integral preserving properties of these subclasses are also discussed.
\end{abstract}

\section{Introduction and preliminaries}

Let $\mathcal{A}_{p}$ denote the class of functions $f(z)$ of the form

$$
f(z)=z^{p}+\sum_{n=1}^{\infty} a_{n} z^{p+n}, \quad(p \in \mathbb{N}=\{1,2,3, \ldots\}),
$$

which are analytic and $p$-valent in the open unit disk $\mathbb{U}=\{z: z \in C$ and $|z|<1\}$. For functions $f$ given by (1) and $g$ given by

$$
g(z)=z^{p}+\sum_{n=1}^{\infty} b_{n} z^{p+n}
$$

the Hadamard product (or convolution) of $f$ and $g$ is defined by

$$
(f * g)(z)=z^{p}+\sum_{n=1}^{\infty} a_{n} b_{n} z^{p+n} .
$$

Given two functions $f$ and $g$, which are analytic in $\mathbb{U}$, the function $f$ is said to be subordinate to $g$ in $\mathbb{U}$ if there exists a function $w$ analytic in $\mathbb{U}$ with

$$
w(0)=0, \quad|w(z)|<1 \quad(z \in \mathbb{U}),
$$

such that

$$
f(z)=g(w(z)) \quad(z \in \mathbb{U})
$$

Received July 23, 2008; Revised January 21, 2009.

2000 Mathematics Subject Classification. 30C45, 30C50, 30C75.

Key words and phrases. multivalent function, convex univalent function, starlike with respect to symmetric points, Hadamard product, subordination, integral operator.

(C)2009 The Korean Mathematical Society 
We denote this subordination by $f(z) \prec g(z)$. Furthermore, if the function $g$ is univalent in $\mathbb{U}$, then $f(z) \prec g(z)(z \in \mathbb{U}) \Longleftrightarrow f(0)=g(0)$ and $f(\mathbb{U}) \subset g(\mathbb{U})$.

Let $\mathcal{P}$ denote the class of analytic functions $h(z)$ with $h(0)=1$, which are convex and univalent in $\mathbb{U}$ and for which $\mathfrak{R}\{h(z)\}>0(z \in \mathbb{U})$.

Analogous to the operator defined recently by Selvaraj and Santhosh Moni [6], we define an operator $D_{\lambda, g}^{\delta} f$ on $\mathcal{A}_{p}$ as follows:

For a fixed function $g \in \mathcal{A}_{p}$ given by

$$
g(z)=z^{p}+\sum_{n=1}^{\infty} b_{n} z^{p+n}, \quad\left(b_{n} \geq 0 ; p \in \mathbb{N}=\{1,2,3, \ldots\}\right),
$$

$D_{\lambda, g}^{\delta} f(z): \mathcal{A}_{p} \longrightarrow \mathcal{A}_{p}$ is defined by

$$
\begin{aligned}
& D_{\lambda, g}^{0} f(z)=(f * g)(z), \\
& D_{\lambda, g}^{1} f(z)=(1-\lambda)(f * g)(z)+\frac{\lambda}{p} z((f * g)(z))^{\prime}, \\
& D_{\lambda, g}^{\delta} f(z)=D_{\lambda, g}^{1}\left(D_{\lambda, g}^{\delta-1} f(z)\right) .
\end{aligned}
$$

If $f(z) \in \mathcal{A}_{p}$, then we have

$$
D_{\lambda, g}^{\delta} f(z)=z^{p}+\sum_{n=1}^{\infty}\left(1+\frac{\lambda n}{p}\right)^{\delta} a_{n} b_{n} z^{p+n},
$$

where $\delta \in \mathbb{N}_{0}=\mathbb{N} \cup\{0\}$ and $\lambda \geq 0$. It easily follows from (3) that

$$
\frac{\lambda z}{p}\left(D_{\lambda, g}^{\delta} f(z)\right)^{\prime}=D_{\lambda, g}^{\delta+1} f(z)-(1-\lambda) D_{\lambda, g}^{\delta} f(z) .
$$

Throughout this paper, we assume that $p, k \in \mathbb{N}, \varepsilon_{k}=\exp \left(\frac{2 \pi i}{k}\right)$, and

$$
f_{p, k}^{\delta}(\lambda ; g ; z)=\frac{1}{k} \sum_{j=0}^{k-1} \varepsilon_{k}^{-j p}\left(D_{\lambda, g}^{\delta} f\left(\varepsilon_{k}^{j} z\right)\right)=z^{p}+\cdots, \quad\left(f \in \mathcal{A}_{p}\right) .
$$

Clearly, for $k=1$, we have

$$
f_{p, 1}^{\delta}(\lambda ; g ; z)=D_{\lambda, g}^{\delta} f(z) .
$$

Making use of the operator $D_{\lambda, g}^{\delta} f(z)$, we now introduce and study the following subclasses of $\mathcal{A}_{p}$ of $p$-valent analytic functions.

Definition. A function $f \in \mathcal{A}_{p}$ is said to be in the class $\mathcal{S}_{p, k}^{\delta}(\lambda ; g ; h)$, if it satisfies

$$
\frac{z\left(D_{\lambda, g}^{\delta} f(z)\right)^{\prime}}{p f_{p, k}^{\delta}(\lambda ; g ; z)} \prec h(z) \quad(z \in \mathbb{U}),
$$

where $h \in \mathcal{P}$ and $f_{p, k}^{\delta}(\lambda ; g ; z) \neq 0(z \in \mathbb{U})$. 
Remark 1.1. If we let

$$
\delta=0 \quad \text { and } \quad g(z)=z^{p}{ }_{l} \mathcal{F}_{m}\left(\alpha_{1}, \ldots, \alpha_{l} ; \beta_{1}, \ldots, \beta_{m} ; z\right),
$$

then $\mathcal{S}_{p, k}^{\delta}(\lambda ; g ; h)$ reduces to the function class $\mathcal{S}_{p, k}^{l, m}\left(\alpha_{1} ; h\right)$ introduced and investigated by Zhi-Gang, Wang Yue-Ping Jiang, and H. M. Srivastava [10].

Remark 1.2. If we let

$$
\delta=0 \quad \text { and } \quad g(z)=z^{p}+\sum_{n=1}^{\infty} \frac{(a)_{n}}{(c)_{n}} z^{p+n},
$$

then $\mathcal{S}_{p, k}^{\delta}(\lambda ; g ; h)$ reduces to the function class $T_{p, k}(a, c ; h)$ introduced and investigated by $\mathrm{N}$-Eng $\mathrm{Xu}$ and Ding-Gong Yang [7].

Remark 1.3. Let

$$
g(z)=h(z)=\frac{1+z}{1-z}
$$

Then $\mathcal{S}_{1,2}^{0}(\lambda ; g ; h)=S_{s}^{*}$. The class $S_{s}^{*}$ of functions starlike with respect to symmetric points has been studied by several authors (see [3], [5], [9]).

Definition. A function $f \in \mathcal{A}_{p}$ is said to be in the class $\mathcal{K}_{p, k}^{\delta}(\lambda ; g ; h)$, if it satisfies

$$
\frac{z\left(D_{\lambda, g}^{\delta} f(z)\right)^{\prime}}{p \varphi_{p, k}^{\delta}(\lambda ; g ; z)} \prec h(z) \quad(z \in \mathbb{U})
$$

for some $\varphi(z) \in \mathcal{S}_{p, k}^{\delta}(\lambda ; g ; h)$, where $h \in \mathcal{P}$ and $\varphi_{p, k}^{\delta}(\lambda ; g ; z) \neq 0$ is defined as in (6).

Definition. A function $f \in \mathcal{A}_{p}$ is said to be in the class $\mathcal{C}_{p, k}^{\delta}(\alpha, \lambda ; g ; h)$, if it satisfies

$$
(1-\alpha) \frac{z\left(D_{\lambda, g}^{\delta} f(z)\right)^{\prime}}{p \varphi_{p, k}^{\delta}(\lambda ; g ; z)}+\alpha \frac{\left(z\left(D_{\lambda, g}^{\delta} f(z)\right)^{\prime}\right)^{\prime}}{p\left(\varphi_{p, k}^{\delta}(\lambda ; g ; z)\right)^{\prime}} \prec h(z) \quad(z \in \mathbb{U})
$$

for some $\alpha(\alpha \geq 0)$ and $\varphi(z) \in \mathcal{S}_{p, k}^{\delta}(\lambda ; g ; h)$, where $h \in \mathcal{P}$ and $\left(\varphi_{p, k}^{\delta}(\lambda ; g ; z)\right)^{\prime} \neq$ 0 .

We need the following lemmas to derive our results.

Lemma $1.4([1])$. Let $\beta(\beta \neq 0)$ and $\gamma$ be complex numbers and let $h(z)$ be analytic and convex univalent in $\mathbb{U}$ with $\mathfrak{R}\{\beta h(z)+\gamma\}>0(z \in \mathbb{U})$. If $q(z)$ is analytic in $\mathbb{U}$ with $q(0)=h(0)$, then the subordination

$$
q(z)+\frac{z q^{\prime}(z)}{\beta q(z)+\gamma} \prec h(z) \quad(z \in \mathbb{U})
$$

implies that

$$
q(z) \prec h(z) \quad(z \in \mathbb{U}) .
$$


Lemma $1.5([2])$. Let $h(z)$ be analytic and convex univalent in $\mathbb{U}$ and let $w(z)$ be analytic in $\mathbb{U}$ with $\mathfrak{R}\{w(z)\} \geq 0(z \in \mathbb{U})$. If $q(z)$ is analytic in $\mathbb{U}$ with $q(0)=h(0)$, then the subordination

$$
q(z)+w(z) z q^{\prime}(z) \prec h(z) \quad(z \in \mathbb{U})
$$

implies that

$$
q(z) \prec h(z) \quad(z \in \mathbb{U}) .
$$

Lemma 1.6. Let $f(z) \in \mathcal{S}_{p, k}^{\delta}(\lambda ; g ; h)$. Then

$$
\frac{z\left(f_{p, k}^{\delta}(\lambda ; g ; z)\right)^{\prime}}{p f_{p, k}^{\delta}(\lambda ; g ; z)} \prec h(z) \quad(z \in \mathbb{U}) .
$$

Proof. For $f(z) \in \mathcal{A}_{p}$, we have from (6) that

$$
\begin{aligned}
f_{p, k}^{\delta}\left(\lambda ; g ; \varepsilon_{k}^{j} z\right) & =\frac{1}{k} \sum_{m=0}^{k-1} \varepsilon_{k}^{-m p} D_{\lambda, g}^{\delta} f\left(\varepsilon_{k}^{m+j} z\right) \\
& =\frac{\varepsilon_{k}^{j p}}{k} \sum_{m=0}^{k-1} \varepsilon_{k}^{-(m+j) p} D_{\lambda, g}^{\delta} f\left(\varepsilon_{k}^{m+j} z\right) \\
& =\varepsilon_{k}^{j p} f_{p, k}^{\delta}(\lambda ; g ; z), \quad(j \in\{0,1, \ldots, k-1\})
\end{aligned}
$$

and

$$
\left(f_{p, k}^{\delta}(\lambda ; g ; z)\right)^{\prime}=\frac{1}{k} \sum_{j=0}^{k-1} \varepsilon_{k}^{j(1-p)}\left(D_{\lambda, g}^{\delta} f\left(\varepsilon_{k}^{j} z\right)\right)^{\prime}
$$

Hence

$$
\begin{aligned}
\frac{z\left(f_{p, k}^{\delta}(\lambda ; g ; z)\right)^{\prime}}{p f_{p, k}^{\delta}(\lambda ; g ; z)} & =\frac{1}{k} \sum_{j=0}^{k-1} \frac{\varepsilon_{k}^{j(1-p)} z\left(D_{\lambda, g}^{\delta} f\left(\varepsilon_{k}^{j} z\right)\right)^{\prime}}{p f_{p, k}^{\delta}(\lambda ; g ; z)} \\
& =\frac{1}{k} \sum_{j=0}^{k-1} \frac{\varepsilon_{k}^{j} z\left(D_{\lambda, g}^{\delta} f\left(\varepsilon_{k}^{j} z\right)\right)^{\prime}}{p f_{p, k}^{\delta}\left(\lambda ; g ; \varepsilon_{k}^{j} z\right)} \quad(z \in \mathbb{U}) .
\end{aligned}
$$

Since $f(z) \in \mathcal{S}_{p, k}^{\delta}(\lambda ; g ; h)$, we have

$$
\frac{\varepsilon_{k}^{j} z\left(D_{\lambda, g}^{\delta} f\left(\varepsilon_{k}^{j} z\right)\right)^{\prime}}{p f_{p, k}^{\delta}\left(\lambda ; g ; \varepsilon_{k}^{j} z\right)} \prec h(z) \quad \text { for } \quad j \in\{0,1, \ldots, k-1\} \text {. }
$$

Noting that $h(z)$ is convex univalent in $\mathbb{U}$, from(11) and (12) we conclude that (10) holds true. 


\section{A set of inclusion relationships}

Theorem 2.1. Let $h(z) \in \mathcal{P}$ with

$$
\mathfrak{R}\{h(z)\}>1-\frac{1}{\lambda} \quad(z \in \mathbb{U} ; \lambda>1) .
$$

If $f(z) \in \mathcal{S}_{p, k}^{\delta+1}(\lambda ; g ; h)$, then $f(z) \in \mathcal{S}_{p, k}^{\delta}(\lambda ; g ; h)$ provided $f_{p, k}^{\delta}(\lambda ; g ; z) \neq 0$ $(z \in \mathbb{U})$

Proof. By using (5) and (6), we have

$(1-\lambda) f_{p, k}^{\delta}(\lambda ; g ; z)+\frac{\lambda z}{p}\left(f_{p, k}^{\delta}(\lambda ; g ; z)\right)^{\prime}=\frac{1}{k} \sum_{j=0}^{k-1} \varepsilon_{k}^{-j p}\left(D_{\lambda, g}^{\delta+1} f\left(\varepsilon_{k}^{j} z\right)\right)=f_{p, k}^{\delta+1}(\lambda ; g ; z)$.

Let $f(z) \in \mathcal{S}_{p, k}^{\delta+1}(\lambda ; g ; h)$ and

$$
w(z)=\frac{z\left(f_{p, k}^{\delta}(\lambda ; g ; z)\right)^{\prime}}{p f_{p, k}^{\delta}(\lambda ; g ; z)} .
$$

Then $w(z)$ is analytic in $\mathbb{U}$, with $w(0)=1$, and from (14) and (15) we have

$$
1-\lambda+\lambda w(z)=\frac{f_{p, k}^{\delta+1}(\lambda ; g ; z)}{f_{p, k}^{\delta}(\lambda ; g ; z)}
$$

Differentiating (16) with respect to $z$ and using (15), we get

$$
w(z)+\frac{z w^{\prime}(z)}{\frac{p}{\lambda}(1-\lambda)+p w(z)}=\frac{z\left(f_{p, k}^{\delta+1}(\lambda ; g ; z)\right)^{\prime}}{p f_{p, k}^{\delta+1}(\lambda ; g ; z)} .
$$

From (17) and Lemma 1.6 we note that

$$
w(z)+\frac{z w^{\prime}(z)}{\frac{p}{\lambda}(1-\lambda)+p w(z)} \prec h(z) \quad(z \in \mathbb{U}) .
$$

In view of (13) and (18), we deduce from Lemma 1.4 that

$$
w(z) \prec h(z) \quad(z \in \mathbb{U}) .
$$

Suppose that

$$
q(z)=\frac{z\left(D_{\lambda, g}^{\delta} f(z)\right)^{\prime}}{p f_{p, k}^{\delta}(\lambda ; g ; z)} .
$$

Then $q(z)$ is analytic in $\mathbb{U}$, with $q(0)=1$, and we obtain from (5) that

$$
f_{p, k}^{\delta}(\lambda ; g ; z) q(z)=\frac{1}{\lambda} D_{\lambda, g}^{\delta+1} f(z)+\left(1-\frac{1}{\lambda}\right) D_{\lambda, g}^{\delta} f(z) .
$$

Differentiating both sides of (20) with respect to $z$, we get

$$
z q^{\prime}(z)+\left(p\left(\frac{1}{\lambda}-1\right)+\frac{z\left(f_{p, k}^{\delta}(\lambda ; g ; z)\right)^{\prime}}{f_{p, k}^{\delta}(\lambda ; g ; z)}\right) q(z)=\frac{z\left(D_{\lambda, g}^{\delta+1} f(z)\right)^{\prime}}{\lambda f_{p, k}^{\delta}(\lambda ; g ; z)} .
$$


Now, we find from (14), (15) and (21) that

$$
q(z)+\frac{z q^{\prime}(z)}{\frac{p}{\lambda}(1-\lambda)+p w(z)}=\frac{z\left(D_{\lambda, g}^{\delta+1} f(z)\right)^{\prime}}{p f_{p, k}^{\delta+1}(\lambda ; g ; z)} \prec h(z) \quad(z \in \mathbb{U}),
$$

since $f(z) \in \mathcal{S}_{p, k}^{\delta+1}(\lambda ; g ; h)$. From (13) and (19) we observe that

$$
\mathfrak{R}\left\{\frac{p}{\lambda}(1-\lambda)+p w(z)\right\}>0 .
$$

Therefore, from (22) and Lemma 1.5 we conclude that

$$
q(z) \prec h(z) \quad(z \in \mathbb{U})
$$

which shows that $f(z) \in \mathcal{S}_{p, k}^{\delta}(\lambda ; g ; h)$.

Theorem 2.2. Let $h(z) \in \mathcal{P}$ with

$$
\mathfrak{R}\{h(z)\}>1-\frac{1}{\lambda} \quad(z \in \mathbb{U} ; \lambda>1) .
$$

If $f(z) \in \mathcal{K}_{p, k}^{\delta+1}(\lambda ; g ; h)$ with respect to $\varphi(z) \in \mathcal{S}_{p, k}^{\delta+1}(\lambda ; g ; h)$, then $f(z) \in$ $\mathcal{K}_{p, k}^{\delta}(\lambda ; g ; h)$ provided $\varphi_{p, k}^{\delta}(\lambda ; g ; z) \neq 0(z \in \mathbb{U})$.

Proof. Let $f(z) \in \mathcal{K}_{p, k}^{\delta+1}(\lambda ; g ; h)$. Then there exists a function $\varphi(z) \in \mathcal{S}_{p, k}^{\delta+1}(\lambda ; g ; h)$ such that

$$
\frac{z\left(D_{\lambda, g}^{\delta+1} f(z)\right)^{\prime}}{p \varphi_{p, k}^{\delta+1}(\lambda ; g ; z)} \prec h(z) \quad(z \in \mathbb{U}) .
$$

An application of Theorem 2.1 yields $\varphi(z) \in \mathcal{S}_{p, k}^{\delta}(\lambda ; g ; h)$ and Lemma 1.6 leads to

$$
\psi(z)=\frac{z\left(\varphi_{p, k}^{\delta}(\lambda ; g ; z)\right)^{\prime}}{p \varphi_{p, k}^{\delta}(\lambda ; g ; z)} \prec h(z) \quad(z \in \mathbb{U}) .
$$

Let

$$
q(z)=\frac{z\left(D_{\lambda, g}^{\delta} f(z)\right)^{\prime}}{p \varphi_{p, k}^{\delta}(\lambda ; g ; z)}
$$

By using (5), $q(z)$ can be written as follows

$$
\varphi_{p, k}^{\delta}(\lambda ; g ; z) q(z)=\frac{1}{\lambda} D_{\lambda, g}^{\delta+1} f(z)+\left(1-\frac{1}{\lambda}\right) D_{\lambda, g}^{\delta} f(z) .
$$

Differentiating both sides of (26) with respect to $z$ and using (14) (with $f$ replaced by $\varphi$ ), we get

$$
q(z)+\frac{z q^{\prime}(z)}{\frac{p}{\lambda}(1-\lambda)+p \psi(z)}=\frac{z\left(D_{\lambda, g}^{\delta+1} f(z)\right)^{\prime}}{p \varphi_{p, k}^{\delta+1}(\lambda ; g ; z)} .
$$


Now, from (24) and (27) we find that

$$
q(z)+\frac{z q^{\prime}(z)}{\frac{p}{\lambda}(1-\lambda)+p \psi(z)} \prec h(z) \quad(z \in \mathbb{U}) .
$$

Combining (23), (25) and (28), we deduce from Lemma 1.5 that

$$
q(z) \prec h(z) \quad(z \in \mathbb{U})
$$

which shows that $f(z) \in \mathcal{K}_{p, k}^{\delta}(\lambda ; g ; h)$ with respect to $\varphi(z) \in \mathcal{S}_{p, k}^{\delta}(\lambda ; g ; h)$.

Corollary 2.3. Let $0<\alpha \leq 1,-1 \leq B<A \leq 1$ and

$$
h(z)=\left(\frac{1+A z}{1+B z}\right)^{\alpha} \quad(z \in \mathbb{U}) .
$$

If $\lambda \leq\left[1-\left(\frac{1-A}{1-B}\right)^{\alpha}\right]^{-1}$, then $\mathcal{S}_{p, k}^{\delta+1}(\lambda ; g ; h) \subset \mathcal{S}_{p, k}^{\delta}(\lambda ; g ; h)$ and $\mathcal{K}_{p, k}^{\delta+1}(\lambda ; g ; h) \subset$ $\mathcal{K}_{p, k}^{\delta}(\lambda ; g ; h)$.

Proof. The analytic function $h(z)$ defined by (29) is convex univalent in $\mathbb{U}$ (see $[8]), h(0)=1$ and $h(\mathbb{U})$ is symmetric with respect to real axis. Thus $h(z) \in \mathcal{P}$ and

$0 \leq\left(\frac{1-A}{1-B}\right)^{\alpha}<\mathfrak{R}\{h(z)\}<\left(\frac{1+A}{1+B}\right)^{\alpha}(z \in \mathbb{U} ; 0<\alpha \leq 1 ;-1<B<A \leq 1)$.

Hence, by using Theorems 2.1 and 2.2 we have the corollary.

Corollary 2.4. Let $0<\alpha \leq 1$ and

$$
h(z)=1+\frac{2}{\pi^{2}}\left(\log \left(\frac{1+\sqrt{\alpha z}}{1-\sqrt{\alpha z}}\right)\right)^{2} \quad(z \in \mathbb{U}) .
$$

If $\lambda \leq \frac{\pi^{2}}{8}(\arctan \sqrt{\alpha})^{-2}$, then $\mathcal{S}_{p, k}^{\delta+1}(\lambda ; g ; h) \subset \mathcal{S}_{p, k}^{\delta}(\lambda ; g ; h)$ and $\mathcal{K}_{p, k}^{\delta+1}(\lambda ; g ; h) \subset$ $\mathcal{K}_{p, k}^{\delta}(\lambda ; g ; h)(z \in \mathbb{U})$.

Proof. The function $h(z)$ defined by (30) is in the class $\mathcal{P}$ (cf. [4]) and satisfies $h(\bar{z})=\overline{h(z)}$. Therefore,

$$
\mathfrak{R}\{h(z)\}>h(-1)=1-\frac{8}{\pi^{2}}(\arctan \sqrt{\alpha})^{2} \geq \frac{1}{2} \quad(z \in \mathbb{U} ; 0<\alpha \leq 1) .
$$

Hence, by Theorems 2.1 and 2.2 we have the desired result.

Theorem 2.5. Let $0 \leq \alpha_{1}<\alpha_{2}$. Then

$$
\mathcal{C}_{p, k}^{\delta}\left(\alpha_{2}, \lambda ; g ; h\right) \subset \mathcal{C}_{p, k}^{\delta}\left(\alpha_{1}, \lambda ; g ; h\right) .
$$

Proof. Let $f(z) \in \mathcal{C}_{p, k}^{\delta}\left(\alpha_{2}, \lambda ; g ; h\right)$. Then there exists a function $\varphi(z) \in \mathcal{S}_{p, k}^{\delta}(\lambda ; g ; h)$ such that

$$
\left(1-\alpha_{2}\right) \frac{z\left(D_{\lambda, g}^{\delta} f(z)\right)^{\prime}}{p \varphi_{p, k}^{\delta}(\lambda ; g ; z)}+\alpha_{2} \frac{\left(z\left(D_{\lambda, g}^{\delta} f(z)\right)^{\prime}\right)^{\prime}}{p\left(\varphi_{p, k}^{\delta}(\lambda ; g ; z)\right)^{\prime}} \prec h(z) \quad(z \in \mathbb{U}) .
$$


Suppose that

$$
q(z)=\frac{z\left(D_{\lambda, g}^{\delta} f(z)\right)^{\prime}}{p \varphi_{p, k}^{\delta}(\lambda ; g ; z)} .
$$

Then $q(z)$ is analytic in $\mathbb{U}$, with $q(0)=1$. Differentiating both sides of $(32)$ we get

$$
q(z)+\frac{\varphi_{p, k}^{\delta}(\lambda ; g ; z)}{\left(\varphi_{p, k}^{\delta}(\lambda ; g ; z)\right)^{\prime}} q^{\prime}(z)=\frac{\left(z\left(D_{\lambda, g}^{\delta} f(z)\right)^{\prime}\right)^{\prime}}{p\left(\varphi_{p, k}^{\delta}(\lambda ; g ; z)\right)^{\prime}}
$$

Now, using (31), (32) and (33) we deduce that

$$
q(z)+w(z) z q^{\prime}(z) \prec h(z)
$$

where

$$
w(z)=\alpha_{2}\left(\frac{z\left(\varphi_{p, k}^{\delta}(\lambda ; g ; z)\right)^{\prime}}{\varphi_{p, k}^{\delta}(\lambda ; g ; z)}\right)^{-1} .
$$

In view of Lemma 1.6 and $\alpha_{2}>0$, we observe that $w(z)$ is analytic in $\mathbb{U}$ and $\mathfrak{R}\{w(z)\}>0$. Consequently, in view of (34), we deduce from Lemma 1.5 that

$$
q(z) \prec h(z) .
$$

Since $0 \leq \frac{\alpha_{1}}{\alpha_{2}}<1$ and since $h(z)$ is convex univalent in $\mathbb{U}$, we deduce from (31) and (35) that

$$
\begin{aligned}
& \left(1-\alpha_{1}\right) \frac{z\left(D_{\lambda, g}^{\delta} f(z)\right)^{\prime}}{p \varphi_{p, k}^{\delta}(\lambda ; g ; z)}+\alpha_{1} \frac{\left(z\left(D_{\lambda, g}^{\delta} f(z)\right)^{\prime}\right)^{\prime}}{p\left(\varphi_{p, k}^{\delta}(\lambda ; g ; z)\right)^{\prime}} \\
= & \frac{\alpha_{1}}{\alpha_{2}}\left(\left(1-\alpha_{2}\right) \frac{z\left(D_{\lambda, g}^{\delta} f(z)\right)^{\prime}}{p \varphi_{p, k}^{\delta}(\lambda ; g ; z)}+\alpha_{2} \frac{\left(z\left(D_{\lambda, g}^{\delta} f(z)\right)^{\prime}\right)^{\prime}}{p\left(\varphi_{p, k}^{\delta}(\lambda ; g ; z)\right)^{\prime}}\right)+\left(1-\frac{\alpha_{1}}{\alpha_{2}}\right) q(z) \\
\prec & h(z) .
\end{aligned}
$$

Thus $f(z) \in \mathcal{C}_{p, k}^{\delta}\left(\alpha_{1}, \lambda ; g ; h\right)$ which completes the proof of Theorem 2.5.

\section{Integral operator}

Theorem 3.1. Let $h(z) \in \mathcal{P}$ and

$$
\mathfrak{R}\{h(z)\}>\max \left\{0,-\frac{\mathfrak{R}(c)}{p}\right\} \quad(z \in \mathbb{U}),
$$

where $c$ is a complex number such that $\mathfrak{R}(c)>-p$. If $f(z) \in \mathcal{S}_{p, k}^{\delta}(\lambda ; g ; h)$, then the function

$$
F(z)=\frac{c+p}{z^{c}} \int_{0}^{z} t^{c-1} f(t) d t
$$

is also in the class $\mathcal{S}_{p, k}^{\delta}(\lambda ; g ; h)$, provided that $F_{p, k}^{\delta}(\lambda ; g ; z) \neq 0(0<|z|<1)$ where $F_{p, k}^{\delta}(\lambda ; g ; z)$ is defined as in $(6)$. 
Proof. Let $f(z) \in \mathcal{S}_{p, k}^{\delta}(\lambda ; g ; h)$. Then from (36) and $\mathfrak{R}(c)>-p$, we note that $F(z) \in \mathcal{A}_{p}$ and

$$
(c+p) D_{\lambda, g}^{\delta} f(z)=c D_{\lambda, g}^{\delta} F(z)+z\left(D_{\lambda, g}^{\delta} F(z)\right)^{\prime} .
$$

Also, from the above, we have

$$
\begin{aligned}
(c+p) f_{p, k}^{\delta}(\lambda ; g ; z) & =\frac{1}{k} \sum_{j=0}^{k-1} \varepsilon_{k}^{-j p}\left(c D_{\lambda, g}^{\delta} F\left(\varepsilon_{k}^{j} z\right)+\varepsilon_{k}^{j} z\left(D_{\lambda, g}^{\delta} F\left(\varepsilon_{k}^{j} z\right)\right)^{\prime}\right) \\
& =c F_{p, k}^{\delta}(\lambda ; g ; z)+z\left(F_{p, k}^{\delta}(\lambda ; g ; z)\right)^{\prime} .
\end{aligned}
$$

Let

$$
w(z)=\frac{z\left(F_{p, k}^{\delta}(\lambda ; g ; z)\right)^{\prime}}{p F_{p, k}^{\delta}(\lambda ; g ; z)} .
$$

Then $w(z)$ is analytic in $\mathbb{U}$, with $w(0)=1$, and from (38) we observe that

$$
p w(z)+c=(c+p) \frac{f_{p, k}^{\delta}(\lambda ; g ; z)}{F_{p, k}^{\delta}(\lambda ; g ; z)} .
$$

Differentiating both sides of (39) with respect to $z$ and using Lemma 1.6, we obtain

$$
w(z)+\frac{z w^{\prime}(z)}{p w(z)+c}=\frac{z\left(f_{p, k}^{\delta}(\lambda ; g ; z)\right)^{\prime}}{p f_{p, k}^{\delta}(\lambda ; g ; z)} \prec h(z) .
$$

In view of (40), Lemma 1.5 leads to $w(z) \prec h(z)$. If we let

$$
q(z)=\frac{z\left(D_{\lambda, g}^{\delta} F(z)\right)^{\prime}}{p F_{p, k}^{\delta}(\lambda ; g ; z)}
$$

then $q(z)$ is analytic in $\mathbb{U}$, with $q(0)=1$, and it follows from $(37)$ that

$$
F_{p, k}^{\delta}(\lambda ; g ; z) q(z)=\frac{c+p}{p} D_{\lambda, g}^{\delta} f(z)-\frac{c}{p} D_{\lambda, g}^{\delta} F(z) .
$$

Differentiating both sides of (41), we get

$$
z q^{\prime}(z)+\frac{z\left(F_{p, k}^{\delta}(\lambda ; g ; z)\right)^{\prime}}{F_{p, k}^{\delta}(\lambda ; g ; z)} q(z)=(c+p) \frac{z\left(D_{\lambda, g}^{\delta} f(z)\right)^{\prime}}{p F_{p, k}^{\delta}(\lambda ; g ; z)}-c \frac{z\left(D_{\lambda, g}^{\delta} F(z)\right)^{\prime}}{p F_{p, k}^{\delta}(\lambda ; g ; z)}
$$

or equivalently,

$$
z q^{\prime}(z)+(p w(z)+c) q(z)=(c+p) \frac{z\left(D_{\lambda, g}^{\delta} f(z)\right)^{\prime}}{p F_{p, k}^{\delta}(\lambda ; g ; z)} .
$$


Now, from (39) and (42) we deduce that

$$
\begin{aligned}
q(z)+\frac{z q^{\prime}(z)}{p w(z)+c} & =\frac{c+p}{p w(z)+c} \frac{z\left(D_{\lambda, g}^{\delta} f(z)\right)^{\prime}}{p F_{p, k}^{\delta}(\lambda ; g ; z)} \\
& =\frac{z\left(D_{\lambda, g}^{\delta} f(z)\right)^{\prime}}{p f_{p, k}^{\delta}(\lambda ; g ; z)} \\
& \prec h(z), \quad \text { because } f(z) \in \mathcal{S}_{p, k}^{\delta}(\lambda ; g ; h) .
\end{aligned}
$$

Combining, $\mathfrak{R}\{h(z)\}>\max \left\{0,-\frac{\mathfrak{R}(c)}{p}\right\}$ and $w(z) \prec h(z)$ we have $\mathfrak{R}\{p w(z)+$ c\} $>0(z \in \mathbb{U})$.

Therefore, from (43) and Lemma 1.5 we find that $q(z) \prec h(z)$, which shows that $F(z) \in \mathcal{S}_{p, k}^{\delta}(\lambda ; g ; h)$.

By applying similar method as in Theorem 3.1, we have:

Theorem 3.2. Let $h(z) \in \mathcal{P}$ and

$$
\mathfrak{R}\{h(z)\}>\max \left\{0,-\frac{\mathfrak{R}(c)}{p}\right\} \quad(z \in \mathbb{U} ; \quad \mathfrak{R}(c)>-p) .
$$

If $f(z) \in \mathcal{K}_{p, k}^{\delta}(\lambda ; g ; h)$ with respect to $\varphi(z) \in \mathcal{S}_{p, k}^{\delta}(\lambda ; g ; h)$, then the function

$$
F(z)=\frac{c+p}{z^{c}} \int_{0}^{z} t^{c-1} f(t) d t
$$

belongs to the class $\mathcal{K}_{p, k}^{\delta}(\lambda ; g ; h)$ with respect to

$$
G(z)=\frac{c+p}{z^{c}} \int_{0}^{z} t^{c-1} g(t) d t
$$

provided that $G_{p, k}^{\delta}(\lambda ; g ; z) \neq 0 \quad(0<|z|<1)$.

Acknowledgement. The authors thank the referees for their valuable comments and helpful suggestions.

\section{References}

[1] S. S. Miller and P. T. Mocanu, On some classes of first-order differential subordinations, Michigan Math. J. 32 (1985), no. 2, 185-195.

[2] _ Differential subordinations and inequalities in the complex plane, J. Differential Equations 67 (1987), no. 2, 199-211.

[3] S. Owa, Z. Wu, and F. Y. Ren, A note on certain subclass of Sakaguchi functions, Bull. Soc. Roy. Sci. Liege 57 (1988), no. 3, 143-149.

[4] F. Rønning, Uniformly convex functions and a corresponding class of starlike functions, Proc. Amer. Math. Soc. 118 (1993), no. 1, 189-196.

[5] K. Sakaguchi, On a certain univalent mapping, J. Math. Soc. Japan 11 (1959), 72-75.

[6] C. Selvaraj and C. Santhosh Moni, Classes of analytic functions of complex order involving a family of generalised linear operators, Pre-print.

[7] N.-E. Xu and D.-G. Yang, Some classes of analytic and multivalent functions involving a liner operator, Mathematical and Computer Modelling (2008), doi: 10.1016/ j.mcm.2008.04.005 - In press. 
[8] _ An application of differential subordinations and some criteria for starlikeness, Indian J. Pure Appl. Math. 36 (2005), no. 10, 541-556.

[9] D.-G. Yang and J.-L. Liu, On Sakaguchi functions, Int. J. Math. Math. Sci. 2003, no. 30, 1923-1931.

[10] Z.-G. Wang, Y.-P. Jiang, and H. M. Srivastava, Some subclasses of multivalent analytic functions involving the Dziok-Srivastava operator, Integral Transforms Spec. Funct. 19 (2008), no. 1-2, 129-146.

Chellian Selvaraj

Department of Mathematics

Presidency College (Autonomous)

Chennai-600 005, India

E-mail address: pamc9439@yahoo.co.in

Kuppathai A. Selvakumaran

Department of Mathematics

R. M. K. EngG. College

KAVARAIPETTAI-601 206, INDIA

E-mail address: selvaa1826@gmail.com 